\title{
Évolution technologique des barrages mobiles sur les voies navigables
}

\section{Technological development of gated weirs on navigable waterways}

\author{
M.A. Petitjean \\ Ingénieur des Ponts et Chaussées \\ au Service Technique Central des Ports Maritimes \\ et des Voies Navigables
}

Les barrages mobiles sur les voies navigables ont toujours pour objet de soutenir la ligne d'eau d'étiage, améliorant ainsi le mouillage naturel de la voie, et donc le tirant d'eau des bateaux, sans aggraver les inondations en crue; dans certains cas, les consignes d'exploitation du barrage permettent de maintenir le tirant d'air au droit de certains ouvrages contraignants pour la navigation (ponts, souterrains), qui constituent alors le point de pivotement du bief jusqu'à ce que la bouchure soit totalement effacée.

Les propos qui suivent sont relatifs aux barrages mobiles sur les voies navigeables de France.

\section{Panorama général de l'évolution technologique}

Les premiers types de barrages mobiles des voies navigables ont été les barrages fixes ou seuils déversants. Cette boutade, qui peut se justifier (un seuil déversant n'est pas autre chose qu'un barrage mobile dont la bouchure est toujours effacée ; de plus, les seuils sont souvent coiffés de rehausses à l'étiage, et ne constituent en général qu'une partie de barrage, d'autres passes étant véritablement mobiles), a surtout pour objet de rappeler que ce type d'ouvrages équipe encore quelques voies navigables, surtout fréquentées aujourd'hui par la navigation de plaisance (bassin de la Maine, réseau breton...).

$\mathrm{La}$ bouchure des premiers barrages véritablement "mobiles" a été constituée de petits éléments : aiguilles appuyées sur des fermettes et un talon du radier (Poirée - 1834), hausses diverses avec arc-boutant, articulées sur le radier ou sur un chevalet, etc. Ces barrages, qui présentaient des avantages constructifs, sont généralement manœuvrés à la main au-dessus du plan d'eau, ce qui rend l'opération souvent pénible et parfois dangereuse. Aussi s'oriente-t-on vers leur reconstruction pro- gressive, au rythme de quelques unités par an, sous forme de barrages à grands éléments qui seront examinés plus loin. L'échéance assez lointaine de la disparition de ces barrages à petits éléments (il en subsiste encore environ 200) impose d'y développer à court terme la sécurité de mancuvre; cet objectif est atteint par des consignes de sécurité (par exemple obligation faite aux agents de s'attacher à un câble de sécurité), par des modifications des moyens de manœuvre (transporteur d'aiguilles, nacelle suspendue à une passerelle...) ou même par un remplacement de bouchure sans reconstruction du barrage (en général, clapets, pour des passes étroites; pour une passe large, un projet de bouchure gonflable est à l'étude).

Depuis la fin du siècle dernier, l'évolution technologique a orienté en général les ingénieurs vers l'utilisation de bouchures à grands éléments; il faut mentionner comme exception à cette règle les barrages à hausses Aubert, manœuvrés d'abord depuis une passerelle supérieure par un chariot à bras, et tout dernièrement (barrage de Denouval, sur la Seine) par des vérins aval, sans superstructure apparente. Les grands éléments ont été divers; citons en particulier ceux manœuvrés par la chute (vanne toît, vanne secteur...) ce qui évitait de disposer sur place d'une source d'énergie. Les types les plus construits actuellement sont les clapets et les segments, les vannes levantes ayant été très utilisées jusqu'à il y a une dizaine d'années.

\section{Les bouchures : clapets et segments}

Les raisons du choix des bouchures à clapets et à segments résident dans leur fiabilité, leur facilité de manœuvre, le peu d'entretien qu'elles exigent, et l'économie de moyens qu'elles permettent (génie civil et métal). 


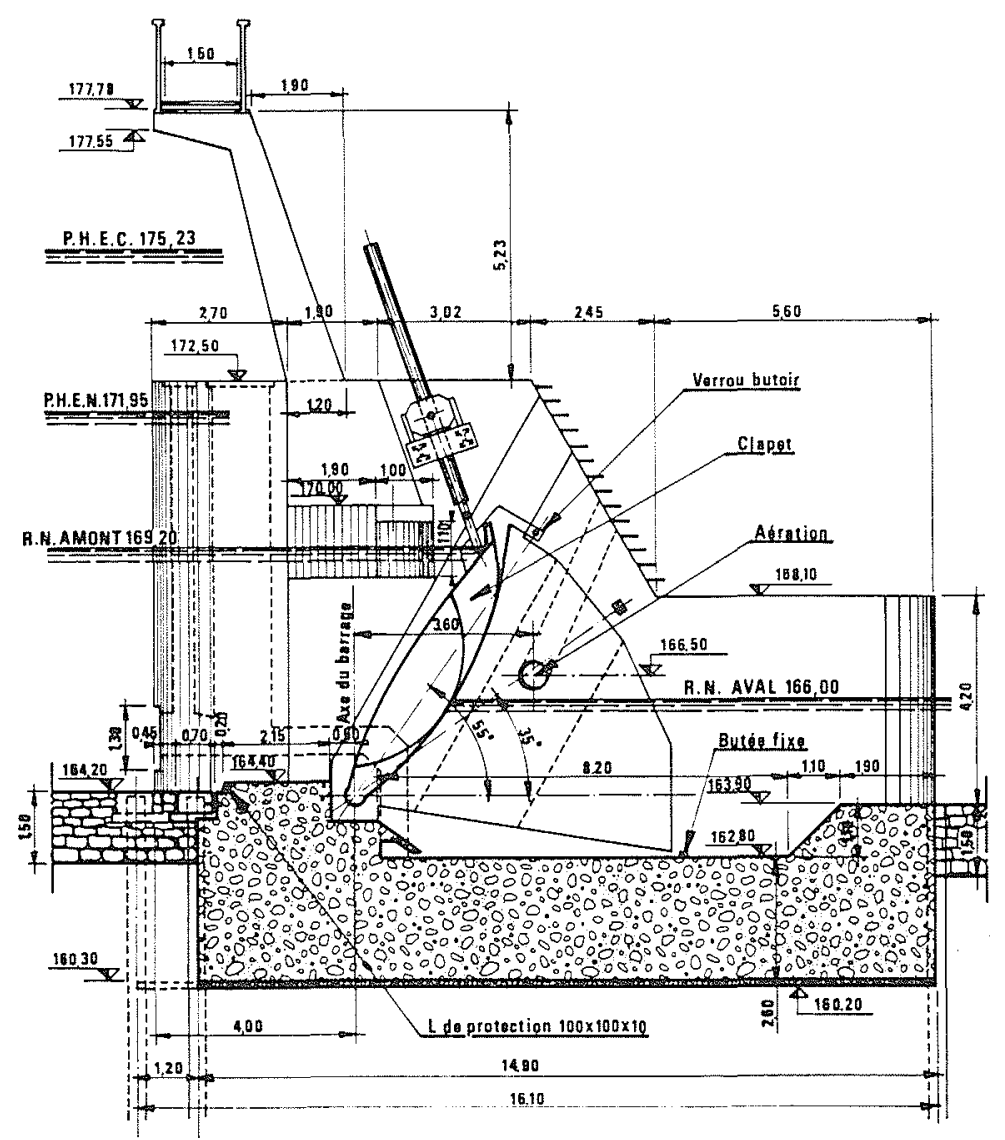

Figure 1 - Vanne clapet : barrage de Thoissey (Saône).

\section{Vannes clapets}

Le clapet est une vanne dont la forme rappelle celle d'une aile d'avion ; il est articulé à sa partie inférieure sur le radier en plusieurs points par des paliers; l'écoulement se fait par déversement supérieur; en position effacée, le clapet est couché dans un encuvement du radier. La manœuvre s'effectue par des vérins (à simple effet) ou par des chaînes (mues par treuils électriques) attaquant le sommet du clapet soit d'un seul côté, soit plus souvent des deux côtés. Le clapet est économique jusqu'à des portées de $30 \mathrm{~m}$ et des hauteurs de bouchure de $5 \mathrm{~m}$ environ.

Outre son prix, le clapet présente deux avantages : tout d'abord, les efforts sont reportés au génie civil à l'amont du barrage, ce qui facilite la stabilité. Ensuite et surtout, l'écoulement de l'eau se faisant en partie supérieure, le niveau du bief a tendance à s'autoréguler.

Les inconvénients des clapets sont les suivants:

- il s'agit de vannes naturellement peu rigides, ce qui oblige à prendre garde aux problèmes de vibrations engendrées par la lame d'eau déversante ("couteaux" au sommet de la vanne), et surtout au problème de synchronisation des organes de manœuvre attaquant la vanne de chaque côté. Avec des vérins, la synchronisation peut être obtenue par des diviseurs de débit; avec des treuils, par des dispositifs mécaniques ou électriques. On prévoit généralement un détecteur de gauchissement de la vanne, qui bloque la mancuvre au-delà d'un seuil fixé. La difficulté liée à la torsion de la vanne se retrouve aussi lorsqu'elle n'est pas manœuvrée, mais simplement soutenue par les vérins : dans ce cas, une fuite sur un seul vérin peut provoquer la rupture de la vanne et de ses articulations; aussi est-il bon de concevoir le circuit hydraulique pour que, en cas de fuite, les deux vérins restent à la même pression; il convient également d'avoir un circuit hydraulique absolument "propre" et aussi de concevoir le clapet de manière qu'il résiste à la torsion en n'étant soutenu que d'un seul côté (ce qui oblige à épaissir le profil), soit qu'un seul vérin soit effectivement prévu au projet, soit que l'on prévoie la possibilité de défaillance d'un des deux vérins (le deuxième vérin a alors peu d'intérêt). On peut enfin diminuer fictivement la portée sur laquelle le clapet doit résister à la torsion en reliant entre eux des demi-clapets par des goupilles cisaillables, ce qui permet, par rapport au système précédent, une économie certaine de matière ;

- la vanne reporte en permanence sur les vérins une partie importante de la pression de l'eau, ce qui oblige à avoir des vérins importants maintenus en charge en permanence. On peut prévoir des butées qui viennent verrouiller le clapet dans deux ou trois positions correspondant aux écoulements les plus fréquents : on vient alors faire reposer le clapet sur ces butées, ce qui décharge les vérins : cette disposition est aussi avantageuse pour l'exploitation, car elle permet de limiter les conséquences d'un abaissement accidentel de la vanne : il suffit que, quelle que soit la position de la vanne, la butée immédiatement inférieure soit sortie; le circuit 
électrique doit être conçu pour empêcher toute manœuvre volontaire de la vanne tant que les butées ne sont pas rentrées;

- la vanne et ses articulations sont difficilement visibles, puisqu'en permanence sous l'eau;

- l'encuvement du radier doit rester libre de tout engravement, afin que le clapet puisse y reposer en crue ; à cet égard, les dispositifs de chasse ne sont pas toujours suffisants.

\section{Vannes segments}

Le segment est une vanne dont le bordé est en arc de cylindre circulaire; les efforts de poussée de l'eau sont reportés aux piles à la hauteur de l'axe de ce cylindre, qui est aussi axe de rotation; l'écoulement se fait par dessous la vanne; en position effacée, le segment est relevé au-dessus de la ligne d'eau de crue de projet. La manœuvre s'effectue comme pour un clapet (vérins ou chaînes mus par treuil, d'un seul côté ou des deux côtés). Le segment est intéressant pour des hauteurs de bouchure importantes (jusqu'à 10 ou $15 \mathrm{~m}$ ), mais des portées plus faibles qu'avec les clapets ( $20 \mathrm{~m}$ environ).

Le segment est intéressant par sa grande robustesse, sa facilité d'entretien (axes de rotation hors d'eau à l'étiage et vanne hors d'eau en crue) et de manœuvre (synchronisation parfaite des deux côtés de la vanne non nécessaire; organes de manœuvre ne supportant que

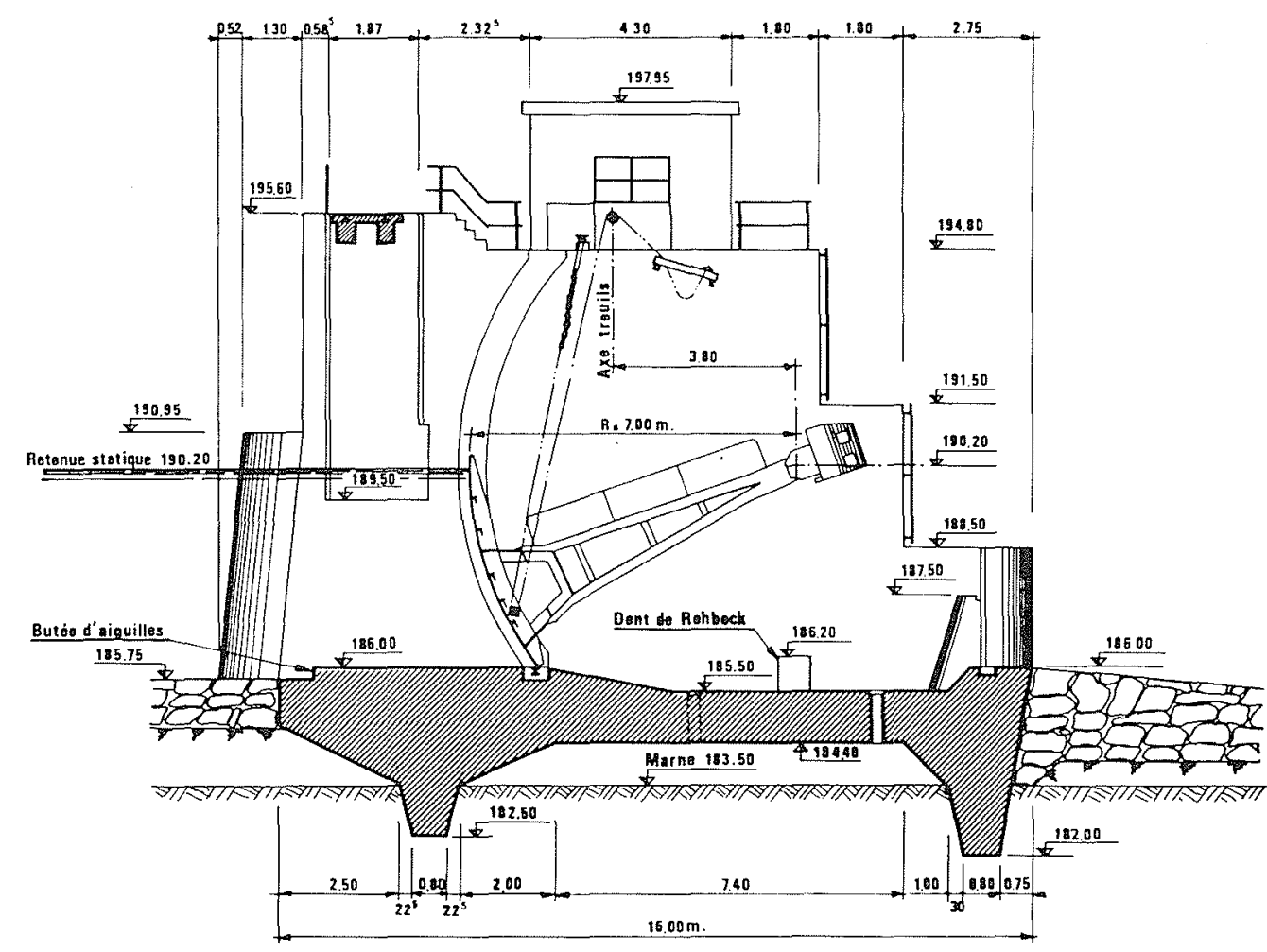

Figure 2 - Vanne segment : barrage de Frouard (Moselle).

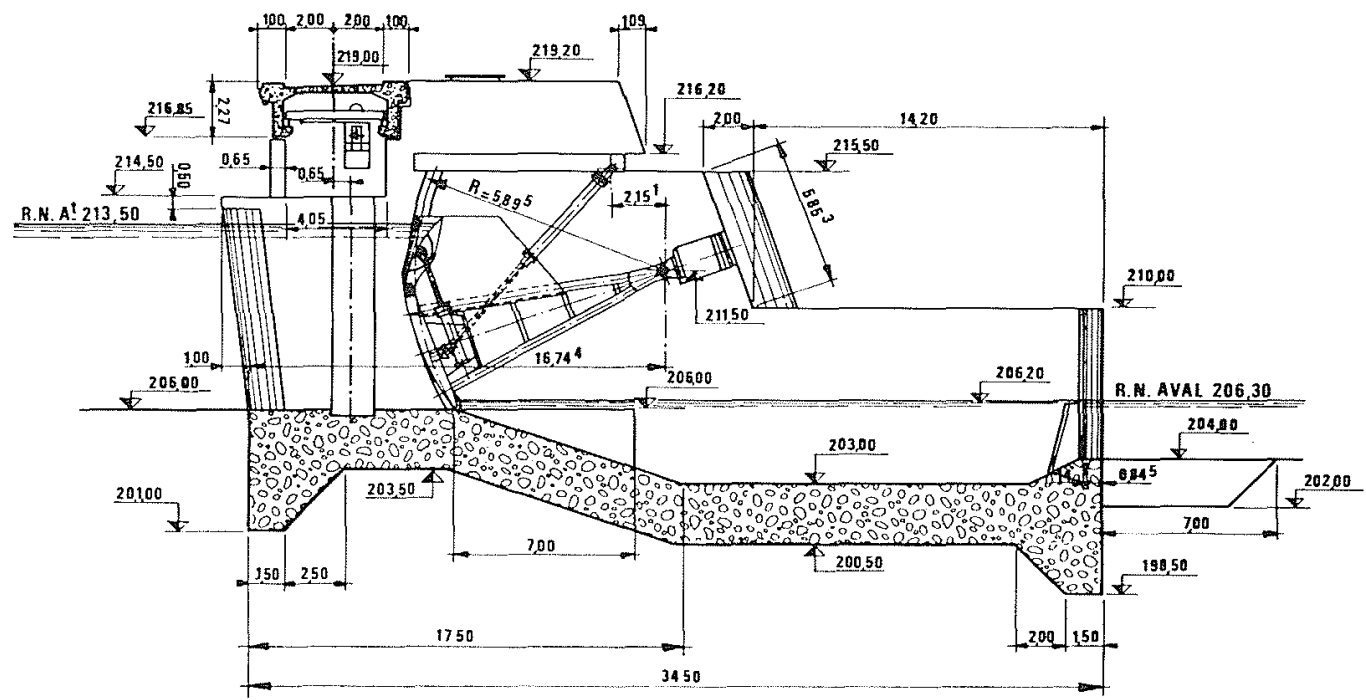

Figure 3 - Vanne segment avec clapet déversant : barrage de Villey-le-Sec (Moselle). 
le poids de la vanne, à l'exclusion des pressions de l'eau), et la simplicité du génie civil du radier (dépourvu de paliers).

Ses inconvénients résident dans le fait que la poussée de l'eau est transmise à l'aval du barrage et uniquement aux piles (ce qui oblige, pour la stabilité d'ensemble, à prévoir un ferraillage important de ces piles), et surtout dans l'écoulement inférieur qui oblige à prévoir dans le radier une importante fosse de dissipation de l'énergie, afin de limiter les érosions à l'aval, et qui fait obstacle à une auto-régulation partielle des niveaux.

Afin de pallier ce dernier inconvénient, le segment est souvent surmonté d'un clapet déversant, qui permet un écoulement supérieur des débits d'étiage, et procure ainsi les avantages d'une auto-régulation.

\section{Autres évolutions technologiques}

\section{Batardeaux de secours}

Le recours à des bouchures à grands éléments a posé le problème des batardeaux de secours, nécessaires pour fermer la passe et mettre la vanne à sec en cas d'incidents. La difficulté réside dans la possibilité de mettre en place le batardeau amont dans un courant. Le batardeau amont peut être constitué d'une vanne complète venant obturer la passe, ou simplement d'une poutre sur laquelle des petits éléments (type aiguilles) viennent trouver leur appui supérieur; vanne ou poutre peuvent être flottantes, et communes à un ensemble d'ouvrages de caractéristiques identiques; si le barrage est surmonté d'un pont, il est possible d'y suspendre la ou les poutres de batardeau. Le batardeau aval pose moins de problèmes, car il est mis en place sans courant; il peut s'agir simplement de profilés verticaux venant s'encastrer dans le radier, et supportant des madriers horizontaux.

\section{Parties fixes}

Le dimensionnement extérieur des parties fixes des barrages mobiles a connu de grands progrès grâce au développement des modèles hydraulique : il est ainsi possible de déterminer la taille du seuil constitué par le radier (modèle mathématique d'ensemble), la longueur et la forme de ce radier, et la longueur de l'aval radier avec la taille des enrochements qui le constituent (modèle physique local). Le rôle de l'aval-radier pour la pérennité de l'ouvrage reste capital, aussi sa constitution (enrochements et couches de transition avec le terrain naturel) et sa mise en œuvre doivent-elles être très étudiées.

Le dimensionnement intérieur du génie civil n'a guère connu d'évolutions récentes; il fait appel au béton armé ; la précontrainte est parfois utilisée pour ancrer le rađier (résistance aux sous-pressions) ou pour recentrer des efforts (poussée de l'eau sur des segments).

\section{Procédés de construction}

Du point de vue de la construction des barrages, l'évolution technologique récente consiste surtout à envisa- ger, pour les vannes de type clapet, la préfabrication à terre d'éléments de radier surmontés de leur bouchure : cette opération, qui a été réalisée pour le barrage de Denouval, présente l'avantage d'éviter le coût et les aléas des travaux sous batardeau en rivière, lorsque l'ouvrage ne peut être construit en site terrestre. Plus classiques sont les techniques de construction en site fluvial à l'abri de batardeaux en palplanches (qui obligent à un phasage soigné des travaux, afin d'éviter le plus possible les risques de submersion du chantier, ou d'aggravation des inondations en crue), ou en site terrestre, à l'abri d'un rabattement de nappe, avec coupure rapide de l'ancien lit de la rivière en fin de travaux.

\section{Automatisation}

L'automatisation des barrages mobiles sur les voies navigables n'est envisageable que sur un ensemble homogène d'ouvrages, présentant tous les mêmes capacités de mancuvre. L'automatisation est relativement simple lorsque le point de pivotement du bief se situe au droit du barrage; les automatisations étaient jusqu'à récemment assez rustiques, et consistaient, lorsque le niveau amont sortait d'une plage fixée, à manœuvrer la vanne pendant une durée fixe suivie d'une période de neutralisation pendant laquelle toute manœuvre était bloquée ; cette disposition avait pour but d'attendre la dissipation des ondes provoquées par le mouvement de la vanne; une automatisation avec utilisation d'un régulateur Raicipe, mis au point par EDF pour les barrages hydroélectriques, a été réalisée, et donne satisfaction depuis deux ans : l'automate déclenche des manœuvres dont la durée et l'espacement sont fonction de la différence entre cote observée et cote de référence, et de la vitesse avec laquelle varie cette différence; on limite les "pompages de la vanne" en augmentant la plage d'insensibilité du système. Il est possible de réaliser des automatisations également dans des cas de point de pivotement éloigné du barrage, mais cela demande une connaissance fine du comportement hydraulique de la rivière, et le recours à une technologie évoluée (microprocesseurs).

La télécommande de barrages mobiles est bien entendu parfaitement envisageable, que ce soit par liaisons filaires ou radio.$$
*
$$

Il apparaît clairement que l'évolution technologique de ces 10 dernières années en matière de barrages mobiles sur les voies navigables françaises a concerné les bouchures, qui ont eu tendance à s'uniformiser autour de deux types principaux à grands éléments (clapets et segments) donnant toute satisfaction à la fois du point de vue de la construction (coût et contraintes sur le génie civil) et de l'exploitation (fiabilité, rapidité de manœuvre, entretien limité). Les autres techniques impliquées dans la construction et l'exploitation des barrages se sont surtout adaptées à cette évolution, en bénéficiant des acquis d'autres domaines (modèles hydrauliques, électromécanique...). 